\title{
The diagnostic value of optical coherence tomography angiography in diabetic retinopathy: a systematic review
}

\author{
David Gildea (10)
}

Received: 2 July 2018/Accepted: 4 October 2018/Published online: 31 October 2018

(C) The Author(s) 2018

\begin{abstract}
Purpose Diabetic retinopathy (DR) is one of the leading causes of blindness worldwide. Accurate investigative tools are essential for the early diagnosis and monitoring of the disease. Optical coherence tomography angiography (OCTA) is a recently developed technology that enables visualisation of the retinal microvasculature.

Methods A systematic review of the literature was performed to examine the diagnostic use of OCTA in DR to date. Medline, EMBASE, and Cochrane databases were searched to find relevant studies. Sixty-one original studies were selected for the review.

Results and discussion OCTA has demonstrated the ability to identify microvascular features of DR such as microaneurysms, neovascularisation, and capillary non-perfusion. Furthermore, OCTA is enabling quantitative evaluation of the microvasculature of diabetic eyes. It has demonstrated the ability to detect early microvascular changes, in eyes with or without clinically evident DR. It has also been shown to detect progressive changes in the foveal avascular zone, and
\end{abstract}

D. Gildea

UCL Institute of Ophthalmology, 11-43 Bath St,

London EC1V 9EL, UK

D. Gildea $(\bowtie)$

Mater Misericordiae University Hospital, Dublin,

Republic of Ireland

e-mail: gildead@tcd.ie vascular perfusion density, with worsening severity of disease. It provides three-dimensional visualisation of the individual retinal vascular networks and is thereby enhancing our understanding of the role of the deeper vasculature in the pathogenesis of diabetic retinopathy and maculopathy.

Conclusion However, limitations exist with current OCTA technology, in respect to the small field of view, image quality, projection artefact, and inaccuracies in analysis of the deeper vascular layers. While questions remain regarding its practical applicability in its present form, with continuing development and improvement of the technology, the diagnostic value of OCTA in diabetic retinopathy is likely to become evident.

Keywords Optical coherence tomography angiography · OCT angiography · OCTA · OCT-A · Diabetic retinopathy - Diabetic maculopathy · Diabetes

\section{Introduction}

Diabetic retinopathy (DR) is one of the leading causes of blindness worldwide [1]. It is the most common cause of blindness in the working population in developed countries [1]. The prevalence of DR is expected to increase due to current diet and exercise 
behaviours [2]. Accurate investigative tools are increasingly important for the diagnosis and monitoring of DR. Fluorescein angiography (FA) is currently the gold standard for the investigation of a number of features of DR including microaneurysms, neovascularisation, capillary non-perfusion, and diabetic macular ischaemia. However, FA is a relatively invasive investigation requiring intravenous injection of fluorescein dye and is known to have a number of risks including nausea, vomiting, itching and urticaria, and even anaphylaxis [3].

Optical coherence tomography angiography (OCTA) is a recently developed technology that enables visualisation of the retinal microvasculature and may offer a noninvasive alternative in the investigation of DR. Several OCTA devices are commercially available currently-the spectral-domain (SD) OCTA device RTVue XR Avanti with AngioVue software (Optovue, Inc., Fremont, California, USA) is the most extensively studied to date. This technology uses the split-spectrum amplitude-decorrelation angiography (SSADA) algorithm to detect blood flow in the retinal tissue [4]. Image areas of $3 \mathrm{~mm} \times 3 \mathrm{~mm}$, or $6 \mathrm{~mm} \times 6 \mathrm{~mm}$, can be acquired. Following acquisition of the retinal OCTA image, it is possible to perform segmentation in order to visualise individual retinal vascular layers separately. The AngioVue software enables segmentation of OCTA images into individual retinal vascular layers-the superficial capillary plexus (SCP) encompasses the vasculature within the ganglion cell layer, and the deep capillary plexus (DCP) encompasses the vasculature bracketing either side of the inner nuclear layer (INL) [4]. The SCP is visualised between an inner boundary at $3 \mu \mathrm{m}$ below the internal limiting membrane (ILM) and an outer boundary at $15 \mu \mathrm{m}$ below the inner plexiform layer (IPL) [4]. The DCP is visualised between an inner boundary at $15 \mu \mathrm{m}$ below the IPL and an outer boundary at $70 \mu \mathrm{m}$ below the IPL [4]. Additional OCTA devices, as well as alternative segmentation methods, have also been studied and will be discussed throughout the course of this review.

The purpose of this systematic review was to assess the diagnostic value of optical coherence tomography angiography (OCTA) in diabetic retinopathy.

\section{Methods}

Literature search

A comprehensive search of multiple databases was performed according to the PRISMA guidelines [5]. Medline, EMBASE, and Cochrane libraries were used to search for the following terms: 'optical coherence tomography angiography'/'OCT angiography'/ 'OCTA' and 'diabetic retinopathy'/'diabetic maculopathy'/'diabetic macular ischaemia'/'diabetic macular oedema'/'diabetes', including MeSH terms and synonyms.

Inclusion/exclusion criteria

The search was performed in order to identify all studies in which OCTA imaging had been used in patients with diabetes, diabetic retinopathy, and diabetic maculopathy. OCTA is a relatively recent development in retinal imaging; therefore, studies since 2014 were included. Studies included were limited to those published in English and in peerreviewed journals. The literature review was limited to original studies and, as such, previous review articles were excluded, as were case reports.

\section{Literature review}

Using the search criteria as outlined above, a total of 144 articles were identified. Following an initial review of the abstracts, removal of duplicate studies, and exclusion of papers that did not fit the selection criteria, 61 articles were selected for the full literature review. OCTA has been used to evaluate a variety of microvascular parameters in eyes with DR-namely microaneurysms, neovascularisation, the foveal avascular zone, vessel density/perfusion density, fractal dimensions/vessel spacing/vessel tortuosity, capillary non-perfusion/diabetic macular ischaemia, and diabetic macular oedema. Each of these will be discussed in turn, followed by a discussion on the future directions of OCTA in DR. 


\section{Results and discussion}

\section{Microaneurysms}

Ishibazawa et al. [4] were one of the earliest groups to evaluate OCTA in diabetic retinopathy. It was a prospective pilot study of 47 eyes (of 25 patients) with DR. The RTVue SD-OCTA device captured $3 \mathrm{~mm} \times$ $3 \mathrm{~mm}$ scans centred on the fovea. Microaneurysms (MAs) were identified in each case using FA. OCTA visualised MAs, in both the SCP and DCP, as focally dilated saccular or fusiform capillaries [4]. The findings were promising in that OCTA demonstrated the ability to visualise MAs, in addition to identifying their location within the retinal vasculature-superficial or deep-which is not possible with FA. However, not all MAs present on FA were visualised on OCTA, suggesting that the sensitivity of MA detection may be a limitation of current technology [4].

Couturier et al. [6] also compared OCTA to FA as a method of MA detection, in a retrospective study of 20 eyes (14 patients) with DR. Using the RTVue SDOCTA device, OCTA identified a significantly lower number of MAs compared to FA $(P=0.02)$ [6]. OCTA detected $62 \%$ of MAs that were detected on FA [6]. It is believed that slow blood flow of less than $0.3 \mathrm{~mm}$ per second cannot be detected by the SSADA algorithm [7]. There were a significantly higher number of MAs detected in the deep plexus compared to the superficial plexus (Mean $\{\mathrm{SD}\}$ number by eye; $4.4 \pm 2.1$ vs. $2.9 \pm 2.3, P=0.034)$, suggesting they primarily originate at this level [6]. It was also noted on OCTA that the majority of MAs occurred at the margin of a capillary non-perfusion area, which appears to support the belief that they are an indicator of retinal ischaemia [6].

Parravano et al. [8] investigated the relationship between the appearance of MAs on structural SD-OCT and their detection with OCTA. One hundred and forty-five MAs, from 30 eyes with diabetic retinopathy, were imaged using the Spectralis SD-OCT and the RTVue SD-OCTA devices [8]. SD-OCT classified MAs based on internal reflectivity-hyporeflective MAs were significantly less likely to be visualised on OCTA [8]. 66.7\% of hyporeflective MAs were visualised, compared to $88.9 \%$ of hyperreflective and moderately reflective MAs [8]. The researchers hypothesised that hyporeflective MAs may be more likely to have a blood flow rate below the threshold as described by Tokayer et al. [7]. The ability to concurrently acquire both en-face structural OCT and OCTA images has also been explored by Miwa et al. [9], suggesting increased MA detection using these imaging techniques simultaneously.

The distribution of MAs in eyes with diabetic macular oedema (DMO) has also been investigated $[10,11]$. Hasegawa et al. [10] published a retrospective study of 33 eyes (27 patients) with DMO who had undergone SD-OCTA (RTVue XR Avanti) and structural SD-OCT imaging. Overall, $77.3 \pm 8.1 \%$ of MAs were located in the DCP of eyes with DMO [10]. On focused analysis of the areas of macular oedema, where macular thickness was $>400 \mu \mathrm{m}$, $91.3 \pm 9.1 \%$ of MAs were located in the DCP [10]. The higher proportion of DCP MAs in areas of macular oedema, compared to areas without oedema, was statistically significant $(P<0.001)$ [10].

Salz et al. [12] used a ultrahigh-speed swept-source OCTA (SS-OCTA) prototype device (New England Eye Centre, Boston, Massachusetts) to image the retinal microvasculature of 43 eyes of diabetic patients. FA was performed in 17 of the 43 cases for comparison. In line with previous studies using SDOCTA, SS-OCTA identified less MAs compared to FA (mean \{SD $\} ; 6.4$ (4.0) vs. 10 (6.9)) [12]. However, the intraretinal depth of MAs was identified in all cases using SS-OCTA. Compared with FA, detection of MAs with SS-OCTA had a sensitivity of $85 \%(95 \%$ CI, 53-97) and a specificity of 75\% (95\% CI, 21-98) [12].

\section{Neovascularisation}

Ishibazawa et al. [4] also identified neovascularisation at the disc (NVD) using the RTVue SD-OCTA device. Eleven of the 47 eyes in this study had proliferative diabetic retinopathy (PDR) and $3 \mathrm{~mm} \times 3 \mathrm{~mm}$ superficial OCTA images of the optic disc head were obtained [4]. In one case, a reduction in blood flow within the neovascularisation at the disc (NVD) was seen in an eye that had been treated with anti-VEGF [4]. It was noted, however, that the reduction in blood flow demonstrated by OCTA does not confirm a structural decrease in the NVD [4].

Studies have shown that OCTA has the ability to quantitatively assess neovascularisation in PDR [13-15]. Savastano et al. [14] conducted a small study of 10 eyes (6 patients) with PDR. It demonstrated that 
OCTA (RTVue XR Avanti) can characterise the extent of NVD with greater accuracy compared to FA [14]. This was due to the fact that early leakage of fluorescein during FA obscures parts of the NVD [14]. As OCTA enabled this enhanced delineation of NVD, the area $\left(\right.$ pixel $^{2}$ ) could be manually delineated by observers [14].

A retrospective study by de Carlo et al. [16] evaluated preretinal neovascularisation (NV) in PDR using OCTA. Fifty-two eyes (34 patients) with PDR were imaged using RTVue SD-OCTA [16]. $6 \mathrm{~mm} \times$ $6 \mathrm{~mm}$ scans of the posterior pole were acquired. OCTA segmentation was performed to visualise blood flow above the ILM. Preretinal NV was visualised in the posterior pole of 13 eyes (12 patients) [16]. The retinal vascular layers were assessed in 12 of the 13 cases -1 case was excluded due to poor image quality. The study demonstrated ability of OCTA to not only visualise preretinal $\mathrm{NV}$, but to also identify areas of capillary non-perfusion and IRMA associated with it. In 11 eyes (92\%), NV was overlying a capillary nonperfusion area [16]. In 6 eyes (50\%), it was noted to be adjacent to an intraretinal microvascular abnormality (IRMA) [16]. However, a considerable drawback of current OCTA technology was also observed in this study - the limited field of view $(6 \mathrm{~mm} \times 6 \mathrm{~mm}$ scans) of the posterior pole would not have detected more peripheral neovascularisation [16].

A 2016 study by Ishibazawa et al. [17] more extensively described the morphology of neovascularisation that can be visualised on OCTA. SD-OCTA (RTVue XR Avanti) imaging was performed on 40 eyes (33 patients) with PDR. This group consisted of 20 treatment-naïve eyes (14 patients) and 20 eyes (19 patients) previously treated with panretinal photocoagulation (PRP). Patients previously treated with antiVEGF were not included in the study. Two patterns of NV were noted: 'exuberant vascular proliferation (EVP)' - defined as an irregular proliferation of fine (small calibre) vessels-and 'pruned vascular loops' of filamentous vessels [17]. The EVP pattern was present in $95 \%$ of treatment-naïve eyes, compared to $65 \%$ of previously treated eyes $(P=0.043)$ [17]. In addition, 36 of the eyes were imaged with FA—all NV sites with EVP $(30 / 30 ; 100 \%)$ demonstrated early phase leakage, while the majority of NV sites without EVP $(5 / 6 ; 83 \%)$ demonstrated only faint leakage [17]. Twelve eyes (eight treatment-naïve, four previously treated) underwent PRP during the study period.
OCTA demonstrated pruning of vessels and a reduction of EVP two-month post-treatment. There was also a significant reduction in the mean flow area of new vessels $\left(0.70 \pm 0.70 \mathrm{~mm}^{2}\right.$ to $0.47 \pm 0.43 \mathrm{~mm}^{2}$, $n=12, P=0.019$ ) [17]. These findings suggest that $\mathrm{NV}$ in PDR may be categorised morphologically using OCTA as being with, or without, EVP. EVP may also have a role as an indicator of active neovascularisation.

Singh et al. [18] demonstrated that OCTA can differentiate between optic disc venous collaterals (OVCs) and NVD in retinal vascular disease, including DR. In contrast to NVD which arises from the venous circulation and penetrates the ILM, OVCs develop within the existing vascular network and can be visualised on OCTA as thin, loopy vessels in the radial peripapillary capillary layer [18]. Differentiation is important as it is believed that OVCs may offer protection from ischaemia, in contrast to the intervention required in cases of NVD [18]. A limitation of this study is that just 20 eyes were imaged, and only a portion of these eyes $(8 / 20)$ had DR-other pathologies included branch and central retinal vein occlusions [18]. Further studies are required before conclusions can be drawn on the applicability of this differentiation in DR.

Foveal avascular zone

A number of studies have used OCTA to analyse the foveal avascular zone (FAZ) in diabetic patients [12, 19-34]. Table 1 details the results of FAZ area measurements in the literature to date (Table 1).

Takase et al. [19] demonstrated that eyes of diabetic patients, even without clinical retinopathy (NDR), had significantly larger FAZ areas compared to healthy controls. The OCTA (RTVue XR Avanti) images of 63 eyes were analysed-19 age-matched control eyes, 24 NDR eyes, and 20 eyes with nonproliferative diabetic retinopathy (NPDR) (17 mild NPDR, 3 moderate NPDR) [19]. FAZ area was measured in both the SCP and DCP of each eye, using the ImageJ software-this method involves binarising the images, before manually delineating the FAZ area, which is then measured in $\mathrm{mm}^{2}$ [19]. In the SCP, the mean FAZ area was significantly increased in both the NDR $\left(0.37 \pm 0.07 \mathrm{~mm}^{2}\right)$ and DR groups $\left(0.38 \pm 0.11 \mathrm{~mm}^{2}\right), \quad$ compared to controls $\left(0.25 \pm 0.06 \mathrm{~mm}^{2}\right)(P<0.01)[19]$. In the DCP also, 


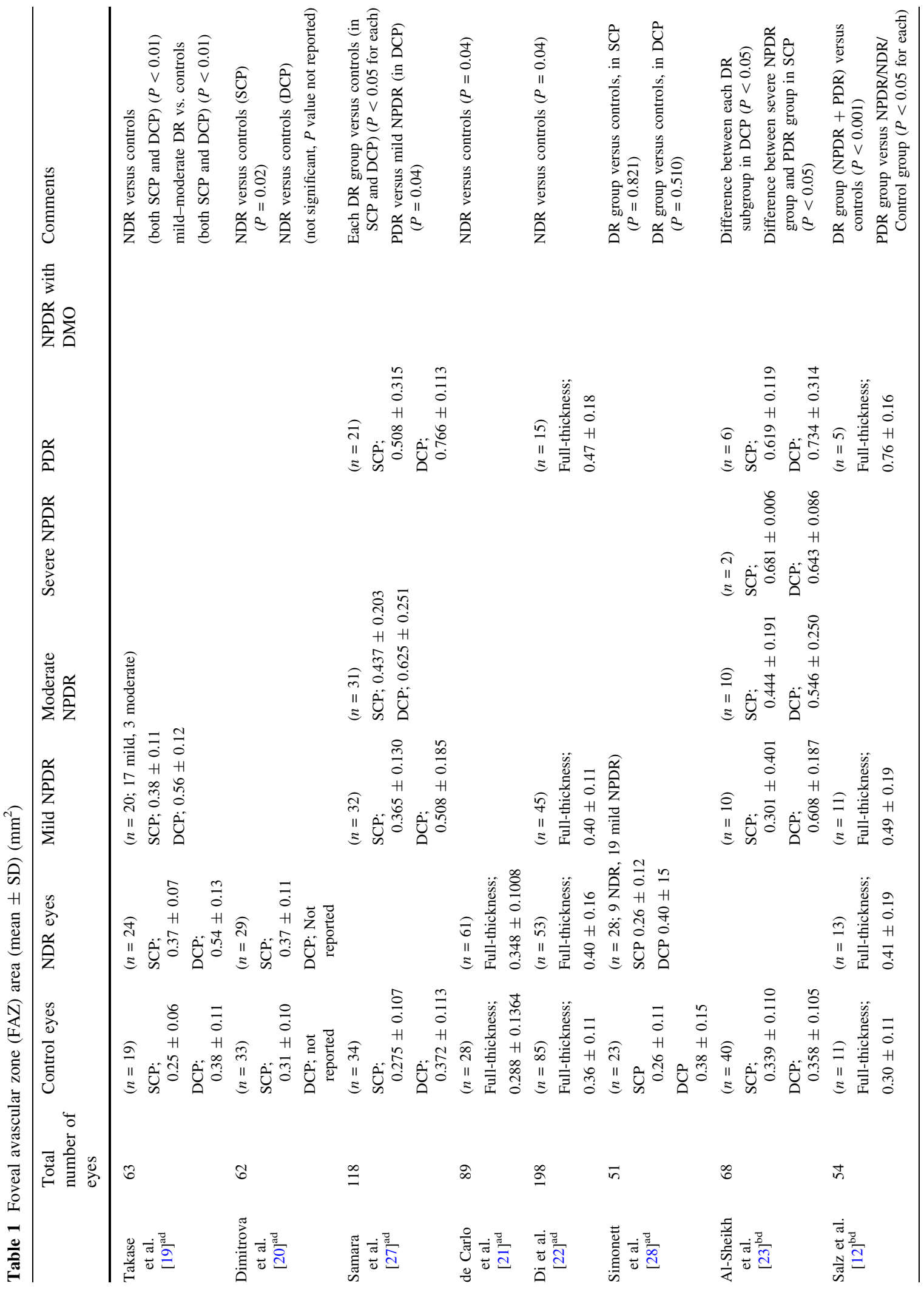




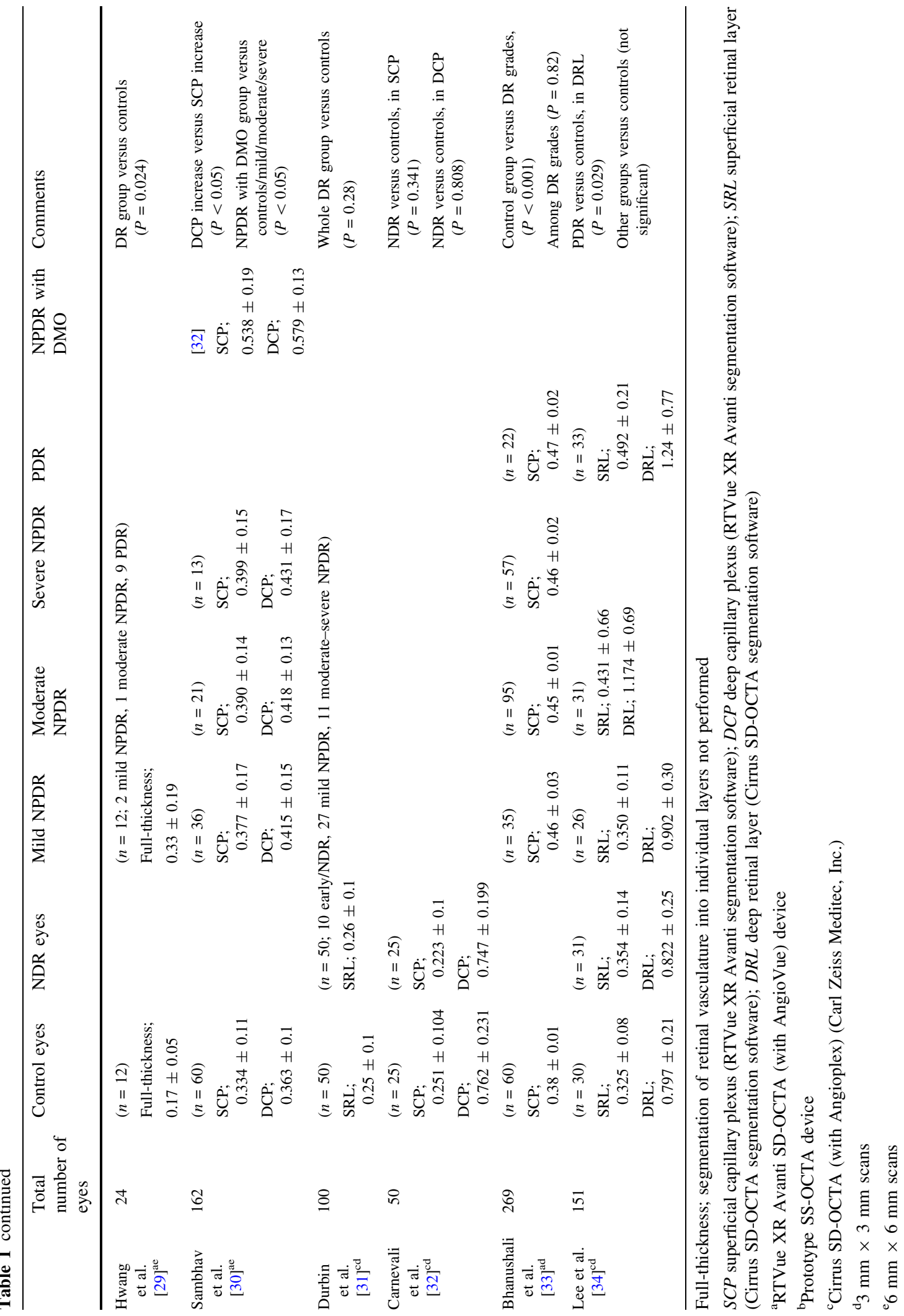


the mean FAZ area was significantly increased in both the NDR $\left(0.54 \pm 0.13 \mathrm{~mm}^{2}\right)$ and DR groups $\left(0.56 \pm 0.12 \mathrm{~mm}^{2}\right), \quad$ compared to controls $\left(0.38 \pm 0.11 \mathrm{~mm}^{2}\right)(P<0.01)$ [19]. There was no significant difference between the NDR and DR groups, possibly due to the mild-moderate NPDR classification of the DR group [19]. These findings suggest that OCTA may be useful for the detection of early microvascular changes, before retinopathy is present on clinical examination.

Dimitrova et al. [20] found the FAZ area to be significantly increased in NDR patients compared to controls in the SCP, but not the DCP. OCTA (RTVue XR Avanti) imaging was performed on 33 control eyes, and 29 NDR eyes. In the SCP, the mean FAZ area was increased in NDR eyes compared to controls $\left(0.37 \pm 0.11 \mathrm{~mm}^{2}\right.$ vs. $\left.0.31 \pm 0.10 \mathrm{~mm}^{2}, P=0.02\right)$ [20]. In the DCP, the mean FAZ area was also increased in NDR eyes; however, this difference was not significant [20].

A prospective study by de Carlo et al. [21] similarly found that OCTA (RTVue XR Avanti) can identify early microvascular alterations in eyes of diabetic patients, before DR is clinically evident. Sixty-one NDR eyes (39 subjects) and 28 control eyes (22 agematched healthy subjects) were imaged [21]. The mean FAZ area was significantly increased in NDR eyes compared to controls $\left(0.348 \pm 0.1008 \mathrm{~mm}^{2}\right.$ vs. $\left.0.288 \pm 0.1364 \mathrm{~mm}^{2}, P=0.04\right)$ [21]. However, the findings are not directly comparable to Takase et al., as full-thickness images of the retinal vasculature were obtained-i.e. SCP/DCP segmentation was not performed.

A larger cross-sectional study by Di et al. [22] also demonstrated increased FAZ area in NDR eyes compared to healthy controls. This study consisted of 85 control eyes (62 healthy subjects) and 113 eyes of diabetic patients (65 patients) — classified using the modified ETDRS system as; 53 NDR eyes, 45 NPDR eyes, and 15 PDR eyes. As with de Carlo et al., fullthickness retinal vasculature images were acquired using OCTA (RTVue XR Avanti). The mean FAZ area was significantly increased in the NDR group compared to controls $\left(0.40 \pm 0.16 \mathrm{~mm}^{2} \quad\right.$ vs. $\left.0.36 \pm 0.11 \mathrm{~mm}^{2}, P=0.04\right)$ [22].

Al-Sheikh et al. [23] used a prototype swept-source OCT device (DRI OCT Triton; Topcon) to acquire OCTA images of eyes with DR and controls. Forty control eyes (20 healthy subjects) and 28 DR eyes (18 patients) were imaged - the DR group consisted of ten mild NPDR eyes, ten moderate NPDR eyes, two severe NPDR eyes, and six PDR eyes [23]. The mean FAZ area was significantly increased in the DR eyes (whole group) compared to controls, in both the SCP and DCP $(P=0.003$, and $P<0.001$, respectively) [23]. On subgroup analysis within the DR group, there was a significant difference in FAZ area between all subgroups at the level of the DCP, and between the severe NPDR and PDR subgroups at the level of the SCP [23] (Table 1).

Additional FAZ parameters have also been investigated using OCTA-including FAZ remodelling measurements, FAZ acircularity and axis ratio, horizontal and vertical FAZ radius, horizontal FAZ diameter, and angle of maximum FAZ diameter.

Krawitz et al. [35] determined that the OCTA parameters of 'acircularity index' (AI) and 'axis ratio' (AR) of FAZ can differentiate between control or NDR eyes, and NPDR or PDR eyes. OCTA (RTVue XR Avanti) images were acquired for 27 age-matched controls and 73 diabetic eyes (16 NDR eyes, 11 mild NPDR eyes, 10 moderate NPDR, 8 severe NPDR eyes, and 28 PDR eyes-graded using the ETDRS system) [35]. FAZ AI was defined as the ratio of the perimeter of the FAZ to the perimeter of a circle of equal areai.e. a perfectly circular FAZ area would have an AI of 1. FAZ AR was defined as the ratio of the major axis to the minor axis of an ellipse generated in the FAZ-i.e. a perfectly circular FAZ would have an axis ratio of 1 . There was a significant increase in both AI and AR in the NPDR and PDR groups compared to the control and NDR groups $(P<0.01$ for each $)$ [35]. On subgroup analysis, a significant difference was also noted in AI between controls and NPDR eyes $(P<0.05)$, and in AR between NDR and mild NPDR eyes $(P<0.01)$ [35].

Di et al. [22] found that the horizontal radius (HR) and vertical radius (VR) of the FAZ were significantly increased in eyes with DR (NPDR and PDR eyes) compared to controls, but no significant difference was observed between NDR eyes and control eyes with these parameters. Freiberg et al. [36] demonstrated that OCTA can be used to distinguish DR eyes from healthy controls based on horizontal FAZ diameter and the angle of maximum FAZ diameter. Twentynine DR eyes and 25 control eyes were imaged using the RTVue XR Avanti OCTA device. The mean horizontal FAZ diameter was significantly larger in 
DR eyes compared to controls in both the SCP $(753 \mu \mathrm{m} \pm 272 \mu \mathrm{m} \quad$ vs. $\quad 573 \mu \mathrm{m} \pm 177 \mu \mathrm{m}$, $P=0.029)$ and DCP $(1009 \mu \mathrm{m} \pm 342 \mu \mathrm{m} \quad$ vs. $659 \mu \mathrm{m} \pm 194 \mu \mathrm{m}, P=0.001)$ [36].

Vessel density/perfusion density

Several OCTA-based methods have been described for measuring the density of vascular perfusion at the macula in eyes with DR- these include capillary perfusion density (CPD), vessel density (VD), flow index (FI), skeleton density (SD), vessel area density (VAD), vessel length density (VLD), and capillary density index (CDI) (Table 2).

A retrospective review by Agemy et al. [37] used OCTA to investigate perfusion density in diabetic eyes. The RTVue OCTA device was used to generate retinal vascular perfusion maps by identifying blood flow [37]. Capillary perfusion density (CPD) was then measured in the SCP, DCP, and choriocapillaris. $3 \mathrm{~mm} \times 3 \mathrm{~mm}$ and $6 \mathrm{~mm} \times 6 \mathrm{~mm}$ scans were obtained in each case. The study included 21 control eyes (12 age-matched subjects), and 56 DR eyes (34 eyes) - the DR group consisted of 11 mild NPDR, 9 moderate NPDR, 9 severe NPDR, and 26 PDR eyes (ETDRS grading) [37]. In all three layers, CPD was found to be significantly reduced in the diabetic group as a whole, compared to controls $(P<0.05$ for each) [37]. A trend of reducing CPD was seen along the spectrum of disease from controls to NPDR to PDR. On subgroup analysis of the $3 \mathrm{~mm} \times 3 \mathrm{~mm}$ scans, mild NPDR patients had significantly lower CPD in both the SCP and DCP compared to controls ( $P<0.05$ for each) [37].

Hwang et al. [29] evaluated the use of automated quantification of vessel density (VD) on OCTA in eyes with DR. This retrospective study used $6 \mathrm{~mm} \times 6$ $\mathrm{mm}$ scans acquired with the RTVue OCTA device and consisted of 12 controls eyes and 12 DR eyes (DRSS grading - two mild NPDR, one moderate NPDR, nine PDR). Segmentation of the individual vascular layers was not performed. The automated quantification technique for VD was obtained by calculating the mean decorrelation signal in eyes of controls-a threshold was defined as 2.3SDs above this mean [29]. The percentage of pixels with flow signal greater than this threshold was used to determine VD. Parafoveal and perifoveal VD were measured. There was a $12.6 \%$ reduction in parafoveal VD $(95 \% \mathrm{CI}, 7.7-17.5 \%$;
$P<0.001)$ and a $10.4 \%$ reduction in perifoveal VD (95\% CI, 6.8-14.1\%; $P<0.001$ ) in DR eyes compared to controls [29]. The findings suggest that this automated technique may be able to differentiate DR eyes from controls.

Sambhav et al. [30] expanded on these findings in a prospective study of 102 newly diagnosed NPDR eyes-classified using the ETDRS system as 36 mild NPDR eyes, 21 moderate NPDR eyes, 13 severe NPDR eyes, and 32 NPDR eyes with DMO. $6 \mathrm{~mm} \times$ $6 \mathrm{~mm}$ scans were obtained using OCTA (RTVue XR Avanti). Sixty control eyes were also imaged for comparison. In contrast to Hwang et al., segmentation of the individual vascular layers was performed using the built-in segmentation software. Vessel density (VD) was defined as the percentage of area occupied by vessels [30]. Flow index (FI) was defined as the average decorrelation value in the segmented area [30]. Analysis was performed on the parafoveal and perifoveal area; however, there were slight differences as to how these areas were defined compared to Hwang et al.- the parafoveal area was defined as a central circular area with diameter $3 \mathrm{~mm}$, excluding the FAZ, while the perifoveal area was defined as a ring area from 3 to $6 \mathrm{~mm}$ from centre of FAZ [30]. In both the SCP and DCP, perfusion indices were found to reduce with increasing severity of DR [30]. On subgroup analysis, each NPDR subgroup significantly differed from control eyes for all parameters-i.e. parafoveal VD and FI, and perifoveal VD and FI, in both the SCP and DCP $(P<0.001)$ [30]. However, the reduction in perfusion indices was significantly more pronounced in the DCP than the SCP $(P<0.05)$ and in the perifoveal region compared to the parafoveal region $(P<0.05)$ [30].

Simonett et al. [28] similarly demonstrated that DCP parafoveal (VD) was significantly reduced in T1DM patients (NDR or mild NPDR) compared to controls $(P<0.001)$; however, the difference was not significant in the SCP. On subgroup analysis, mild NPDR eyes had a significantly reduced DCP parafoveal VD compared to controls $(P<0.001)$ (Table 2$)$ [28]. DCP parafoveal VD was also reduced in NDR eyes compared to controls, but this was not statistically significant $(P=0.052)$ (Table 2) [28]. Direct comparisons with the aforementioned studies may not be appropriate as the definition of the parafoveal area in this study also differed-it was delineated by an annulus between a circle of $1 \mathrm{~mm}$ diameter (centred 


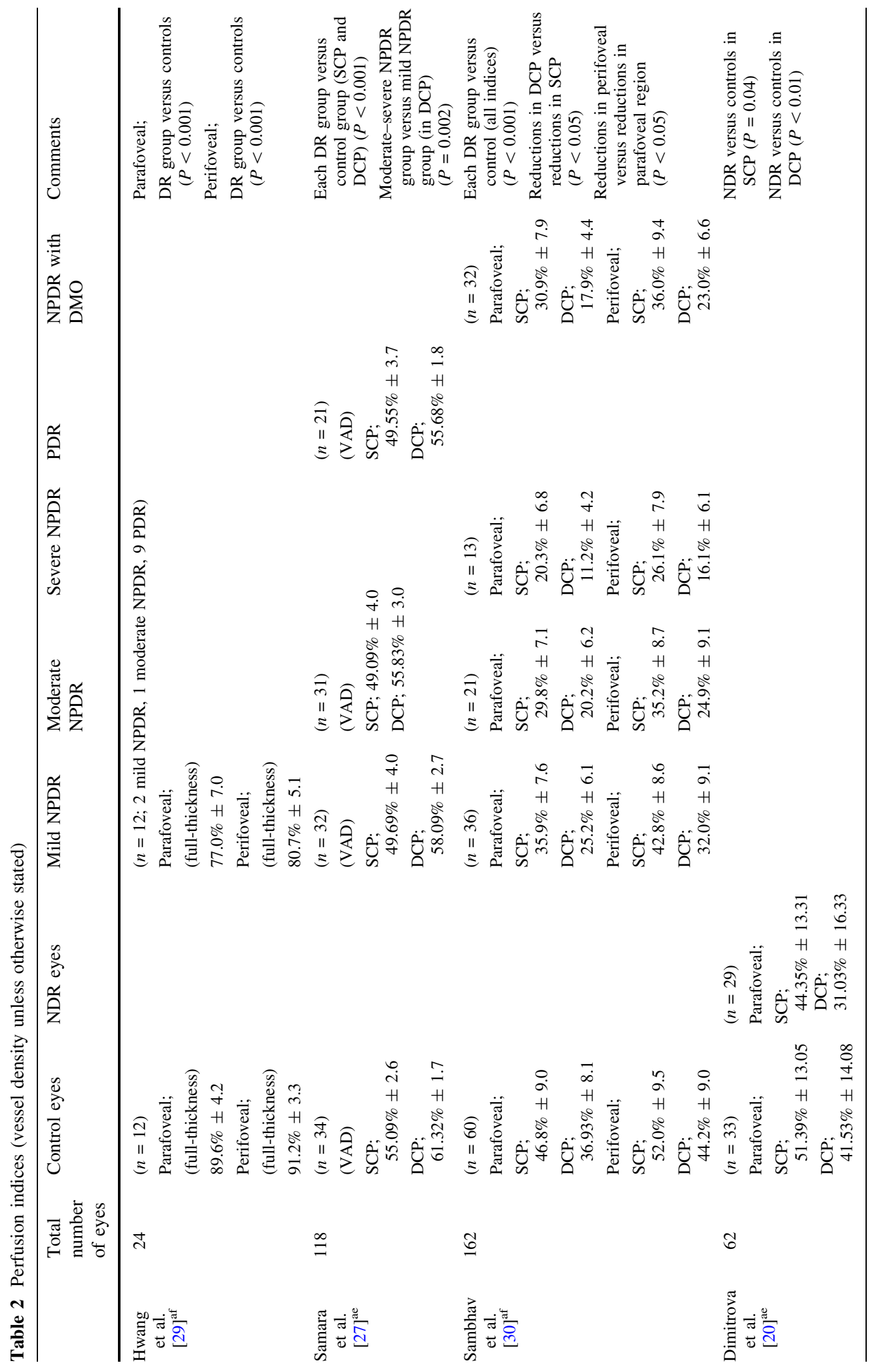




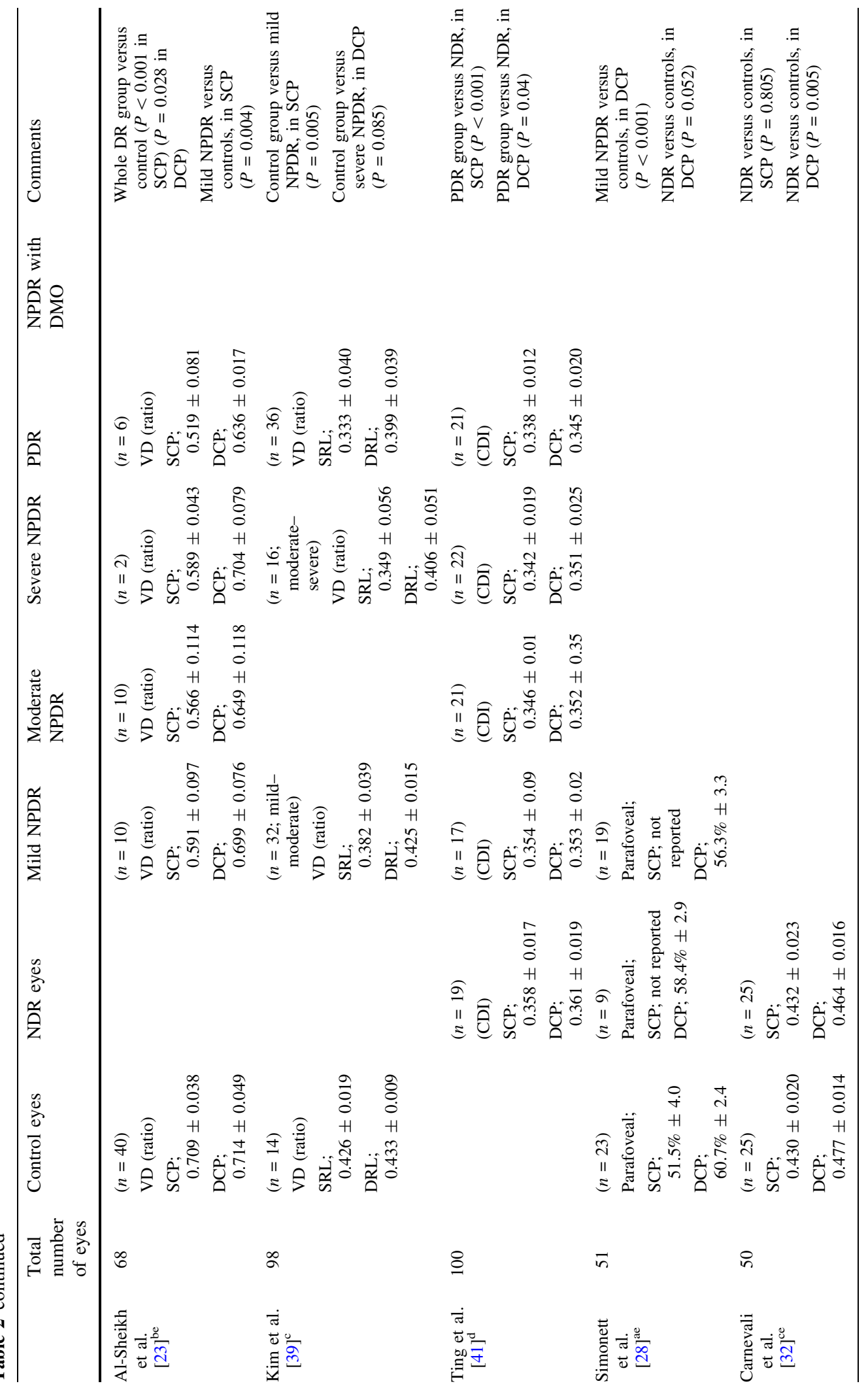




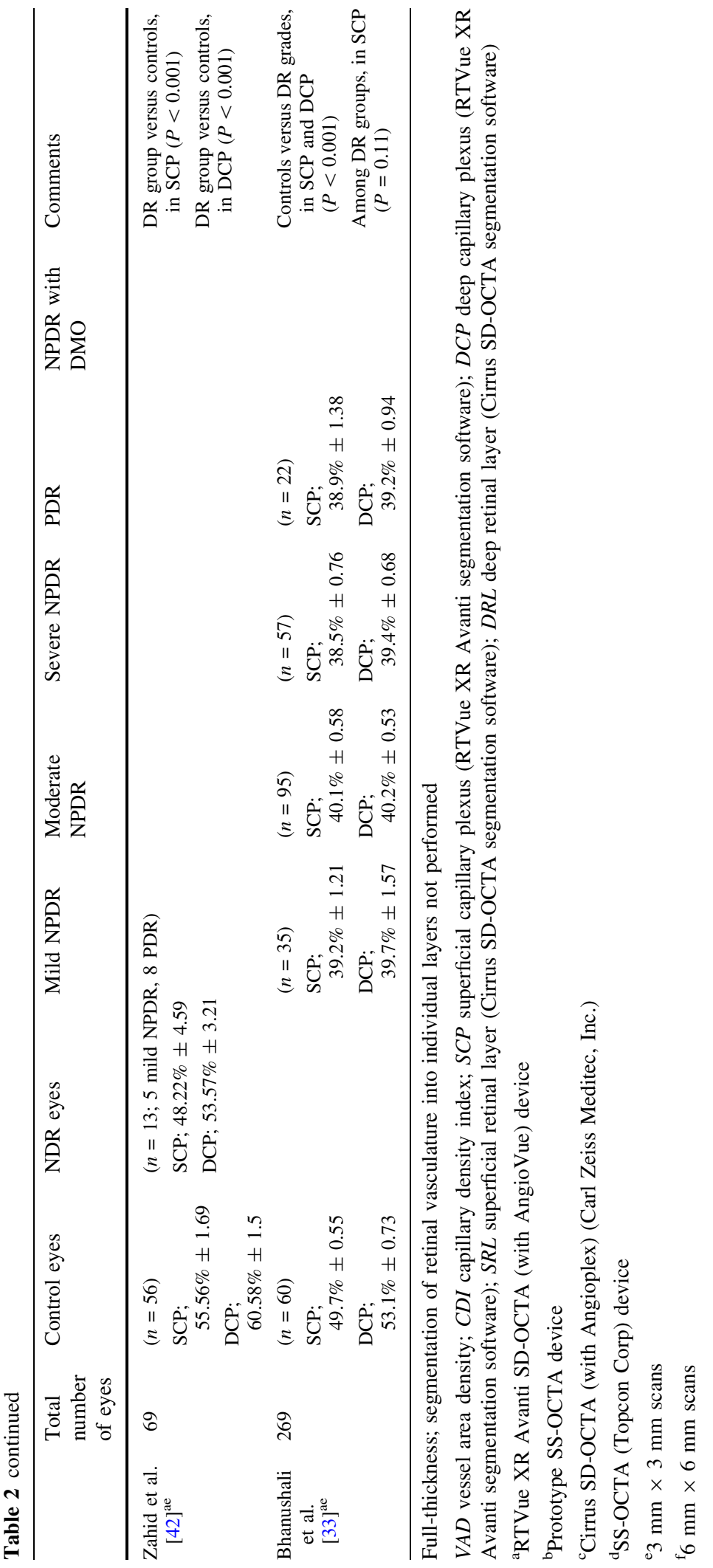


on the fovea) and a circle of $2.5 \mathrm{~mm}$ diameter (also centred on the fovea) [28].

A number of studies have discussed the difficulty in accurately assessing the DCP vasculature using current OCTA technology [20, 23]. Projection artefact, whereby the vascular flow of more superficial vessels can alter the interpretation of deeper vessels, may account for this difference [38]. This may account for some of the inconsistencies observed in relation to DCP perfusion density data, in addition to the previously discussed DCP FAZ measurements.

For example, Dimitrova et al. [20] found that DCP VD was lower than the SCP VD in both control eyes and NDR eyes. SCP VD was significantly reduced in NDR eyes compared to controls $(44.35 \% \pm 13.31 \mathrm{vs.}$ $51.39 \% \pm 13.05, P=0.04$ ) [20]. DCP VD was also significantly reduced in NDR eyes compared to controls $\quad(31.03 \% \pm 16.33$ vs. $41.53 \% \pm 14.08$, $P<0.01$ ) [20]. Al-Sheikh et al. [23] found that mean VD was also significantly lower in both the SCP and DCP in eyes with diabetic retinopathy compared to normal subjects. However in this study, using a prototype SS-OCTA device, a more severe VD reduction was seen in the SCP (Table 2) [23]. Mild NPDR eyes could be differentiated from control eyes in the SCP $(P=0.004)$, but not the DCP $(P=0.544)$ [23].

Kim et al. [39] used the Cirrus SD-OCT with Angioplex (Carl Zeiss Meditec, Dublin, CA, USA) to quantify skeleton density (SD) (a measurement of the length of vessels based on a skeletonised OCTA image), and vessel density (VD) (the proportion of image containing OCTA signal compared to total area imaged). An automated segmentation algorithm was used to produce three slabs - a superficial retinal layer (SRL); ILM to $110 \mu \mathrm{m}$ above the retinal pigment epithelium (RPE) (inner 60\% of retina), a deep retinal layer (DRL); $110 \mu \mathrm{m}$ above RPE to RPE (outer 40\%), and an outer retinal layer; $110 \mu \mathrm{m}$ above RPE to external limiting membrane [39]. The study included 14 control eyes and 84 DR eyes-graded using the ETDRS as; 32 mild-moderate NPDR, 16 moderatesevere NPDR, and 36 PDR eyes. On full-thickness slabs (i.e. non-segmented), there were statistically significant differences in the parameters when comparing healthy eyes with moderate-severe NPDR or PDR eyes and when comparing mild NPDR eyes with moderate-severe or PDR eyes (Table 2) [39]. However, a statistically significant difference between healthy and mild-moderate NPDR was noted only in the SRL (Table 2) [39]. A notable limitation of this study was the number of eyes excluded from the final analysis due to insufficient image quality-116 eyes had been imaged during the defined study period, and 14 of these were excluded for this reason.

Samara et al. [27] also used the RTVue OCTA device to quantitatively analyse VD in eyes with DR. Two methods of VD measurement were used-vessel area density (VAD) and vessel length density (VLD) [27]. In contrast to Hwang et al., $3 \mathrm{~mm} \times 3 \mathrm{~mm}$ scans were used for analysis - and VD measurements were obtained for the entire scan area (unlike para/perifoveal areas) [27, 29]. Similar to Hwang et al., images were binarised using an automated thresholding algorithm, and blood vessels were identified by pixels that had decorrelation values above a threshold $[27,29]$. The FAZ area was excluded. VAD was calculated as the area where vasculature was present(pixels)/(Total area - FAZ area) (pixels) [27]. The image was then skeletonised for measurement of VLD and calculated as - the length of skeletonised vasculature $(\mathrm{mm}) /\left(\right.$ Total area - FAZ area) $\left(\mathrm{mm}^{2}\right)$ [27]. Measurements were obtained for the SCP and DCP. The study consisted of 34 control eyes and 84 DR eyes-the DR group included 32 mild NPDR, 31 moderate-severe NPDR, and 21 PDR eyes [27]. VAD and VLD were significantly reduced in all stages of DR compared to controls in both the SCP and DCP $(P<0.001$ for all) (Table 2) [27]. In the SCP, VAD and VLD did not differ significantly between DR stages [27]. In the DCP, VAD was significantly reduced in the moderate-severe NPDR group compared to the mild NPDR group (55.83\% vs. $58.09 \%$, $P=0.002$ ) and was also significantly reduced in the PDR group compared to the mild NPDR group (55.68\% vs. $58.09 \%, P=0.004)$ [27]. In the DCP, VLD was significantly reduced in the moderatesevere NPDR group compared to the mild NPDR group (20.81 mm $\mathrm{mm}^{-1}$ vs. $\left.22.12 \mathrm{~mm}^{-1}, P=0.015\right)$ and was also significantly reduced in PDR compared to mild NPDR $\left(20.34 \mathrm{~mm}^{-1}\right.$ vs. $22.12 \mathrm{~mm}^{-1}$, $P=0.002$ ) [27]. These findings suggest that the VD parameters, VAD and VLD, may be useful in evaluating the severity of DR.

You et al. [40] suggested that there is good intravisit and intervisit reproducibility of OCTA vessel density measurements in both healthy and diseased eyes. Fifteen control eyes and 22 eyes with retinopathy-16 
of whom had DR without DMO—were imaged using the RTVue OCTA device. Vessel density in the SCP was calculated using the AngioVue software, defined as the proportion of the area occupied by vesselseach patient attended two separate scanning sessions, with two scans at each session. The intravisit coefficient of variation varied from 2.1 to $4.9 \%$ for healthy eyes and 3.4 to $6.8 \%$ for eyes with retinopathy [40]. The intervisit coefficient of variation varied from 2.9 to $5.1 \%$ for healthy eyes and 4.0 to $6.8 \%$ for eyes with retinopathy [40]. However, as DR was not the only retinopathy investigated and eyes with DMO were not included, these findings may not apply to all DR eyes.

Ting et al. [41] investigated capillary density index (CDI) as a parameter for vascular perfusion in DR. The SS-OCTA (Topcon Corp) device was used to image 100 diabetic eyes attending a DR screening clinic at the Singapore National Eye Centre-this consisted of 19 NDR, 17 mild NPDR, 21 moderate NPDR, 22 severe NPDR, and 21 PDR eyes. CDI was calculated using ImageJ software, and a technique in which OCTA images were binarised and the luminal area was measured [41]. CDI analysis was performed in a circle with a radius of $1.5 \mathrm{~mm}$, centred on the fovea. There was a trend of reducing CDI from NDR eyes through to PDR eyes in both the SCP and DCP (Table 2) [41]. There were statistically significant differences between the PDR group and NDR group in both the SCP $(P<0.001)$, and DCP $(P=0.04)$ [41]. The findings demonstrate the potential of CDI as another perfusion index for evaluating the severity of DR; however, larger studies are required to investigate its ability to distinguish less severe disease.

Fractal dimension/vessel spacing/vessel tortuosity

OCTA has also been used to investigate retinal microvasculature in terms of branching complexity, vessel spacing, and vessel tortuosity.

It is hypothesised that a loss of small branching vessels in diseases such as DR, results in a reduced branching complexity of the retinal vasculaturefractal dimensional (FD) analysis can be used as a measurement of branching complexity [39, 42]. A retrospective study by Zahid et al. [42] analysed the OCTA (RTVue XR Avanti) images of 56 control eyes and 13 DR eyes (five mild NPDR and eight PDR eyes). FD was calculated using ImageJ software and a 'boxcounting' method on binarised OCTA images [42].
Mean FD was significantly reduced in DR eyes compared to controls in both the SCP $(1.56 \pm 0.08$ vs. $1.64 \pm 0.05, P=0.005)$ and DCP $(1.60 \pm 0.11$ vs. $1.72 \pm 0.04, P=0.003$ ) [42]. The aforementioned study by Kim et al. [39] similarly demonstrated that there is a progressive reduction in FD with increasing severity of DR. Using the Cirrus SD-OCTA device, 14 control eyes and 84 DR eyes (32 mild NPDR, 16 severe NPDR, 36 PDR) were imaged. In full-thickness, non-segmented slabs, FD was significantly reduced in severe NPDR or PDR eyes compared to controls or mild NPDR eyes [39]. Following analysis of the segmented slabs, FD in the SRL was significantly reduced in mild NPDR eyes compared to controls $\quad(1.695 \pm 0.018 \quad$ vs. $1.717 \pm 0.006$, $P=0.026)$.

Bhanushali et al. [33] investigated the potential value of large vessel spacing as a marker of DR progression. This was a prospective study of 60 controls and 209 DR eyes-graded using the ETDRS system as 35 mild NPDR, 95 moderate NPDR, 57 severe NPDR, and 22 PDR eyes. The RTVue OCTA device was used to measure the spacing between large and small vessels, in the SCP and DCP. Large vessel spacing in the SCP was significantly higher in the severe NPDR and PDR groups compared to mild NPDR and moderate NPDR $(P=0.04)$ [33]. However, small vessel spacing in the SCP was significantly higher in mild NPDR compared to other DR grades $(P<0.001)$ [33].

Lee et al. [34] quantitatively analysed vessel tortuosity (VT) on the Cirrus SD-OCTA images of diabetic eyes. The study consisted of 30 control eyes and 121 diabetic eyes-graded as per the modified Airlie House Classification system as: 31 NDR, 26 mild NPDR, 31 moderate-severe NPDR, and 33 PDR eyes. $3 \mathrm{~mm} \times 3 \mathrm{~mm}$ scans of the SRL and DRL were acquired, within which $3 \mathrm{~mm}$ or $1.5 \mathrm{~mm}$ diameter circular areas were analysed. VT was calculated as the sum of actual branch lengths divided by sum of straight lengths between branch connections, using skeletonised OCTA images [34]. In the SRL, VT trended upwards with progressing DR severity until moderate-severe NPDR [34]. There was no significant increase in VT between healthy eyes and NDR eyes. However, in the SRL $1.5 \mathrm{~mm}$ area scans, VT was significantly increased in mild NPDR compared to healthy eyes and NDR eyes $(P<0.05)$ [34]. In both the $3 \mathrm{~mm}$ and $1.5 \mathrm{~mm}$ area scans, moderate to severe 
NPDR eyes demonstrated significantly higher VT compared to healthy eyes and NDR eyes [34]. In the DRL, similar trends were observed; however, the only statistically significant increase in VT was noted between moderate-severe NPDR and healthy/NDR eyes [34].

Capillary non-perfusion/diabetic macular ischaemia

Ishabazawa et al. [4] demonstrated that areas of retinal non-perfusion can be visualised in eyes with DR using OCTA. Furthermore, these areas can be delineated in both the SCP and DCP and measured in $\mathrm{mm}^{2}$ [4]. However, a limitation of retinal non-perfusion analysis with current OCTA technology was noted in that the small field of view imaged, $3 \mathrm{~mm} \times 3 \mathrm{~mm}$, does not allow for evaluation of more peripheral perfusion abnormalities [4].

Couturier et al. [6] compared OCTA to FA in terms of its ability to identify macular capillary nonperfusion in 20 eyes with DR. $3 \mathrm{~mm} \times 3 \mathrm{~mm}$ OCTA (RTVue XR Avanti) images were compared to magnified $3 \mathrm{~mm} \times 3 \mathrm{~mm}$ early phase FA squares. OCTA identified areas of non-perfusion in the SCP of all eyes, but in the DCP of just $35 \%$ of cases, indicating a difference in the pattern of microvascular change in each plexus [6]. OCTA more accurately delineated areas of capillary non-perfusion outside the FAZ, compared to FA [6]. This was due to the fact that there was superposition of the deep and superficial capillary plexus, as well as some obscuring vessel leakage, with FA imaging.

The ability of OCTA to detect eyes with DR based on capillary non-perfusion was investigated by Hwang et al. [29]. The term, total avascular area (TAA), was used to describe the sum of the avascular (nonperfused) areas in a $6 \mathrm{~mm} \times 6 \mathrm{~mm}$ OCTA (RTVue XR Avanti) image. An automated quantification technique was used to calculate TAA - the mean decorrelation signal in controls was calculated, and areas with flow signal less than 1.2SDs above this mean were identified as being avascular [29]. TAA was significantly increased in DR eyes compared to controls $\left(1.00 \pm 0.27 \mathrm{~mm}^{2}\right.$ vs. $0.18 \pm 0.07 \mathrm{~mm}^{2}$, $P<0.001)$ [29]. The findings suggest that TAA may have a diagnostic role in eyes with DR; however, the small sample size (12 controls, 12 DR eyes) is a limitation of this study. Furthermore, nine of the DR group were graded as PDR. Larger studies with increased numbers of NPDR eyes are required.

OCTA has enabled a greater understanding of the relationship between capillary non-perfusion and diabetic macular ischaemia (DMI). Minnella et al. [43] demonstrated that eyes with DMI had significantly increased perifoveal 'no flow' areas compared to controls, in both the SCP and DCP. A 2016 study by Scarinci et al. found that areas of DCP non-perfusion in eyes with DMI, corresponded precisely with areas of outer retinal disruption on structural OCT imaging [44]. By contrast, eyes with normal DCP perfusion did not exhibit outer retinal structural abnormalities [44].

A number of studies have investigated the ability of OCTA to grade DMI [45-48]. Currently, DMI is graded with FA images using the ETDRS protocols as; absent (no FAZ disruption), questionable (FAZ not smooth/oval, but no clear pathology), mild ( $<$ half FAZ circumference destroyed), moderate ( $>$ half FAZ circumference destroyed), severe (FAZ outline completely destroyed), or ungradable [49].

A 2016 study by Bradley et al. [45] compared OCTA to FA in the grading of DMI. The RTVue OCTA images-SCP, DCP, and choriocapillaris-of 24 DR eyes were retrospectively analysed. The SCP OCTA images were graded using the ETDRS protocols [49] and compared to FA images. The DCP and choriocapillaris OCTA images were graded with a newly devised system [45]. In the DCP, this grading was absent (no disruption of FAZ), questionable (FAZ not smooth/oval, but no clear pathology), mild/moderate (FAZ disrupted in $\leq 2$ quadrants), severe (FAZ disrupted in $\geq 3$ quadrants), or ungradable (poor image quality, artefact) [45]. In the choriocapillaris, it was graded as ischaemia present (loss of speckled hyperreflectance, or dark defects), ischaemia absent, or ungradable [45]. Two masked graders independently assessed the images. There was moderate agreement between DMI grading with the FA and OCTA images of the SCP (intragrader weighted $\kappa$ values; 0.53 for grader $1,0.41$ for grader 2) - there was no difference in DMI grade in $60.4 \%$ of eyes, $33.3 \%$ had a difference of one grade, $2.1 \%$ had a difference of two grades, and $4.2 \%$ were ungradable [45]. In terms of reproducibility of this OCTA-based grading, there was substantial intergrader agreement in terms of the DMI grade acquired using OCTA for the SCP, DCP, and choriocapillaris (weighted $\kappa$ values; 0.65, 0.61, and 0.65 , respectively) [45]. While larger, prospective 
trials are required to validate these findings, they suggest that OCTA may provide an alternative to FA in the investigation of DMI.

\section{Diabetic macular oedema}

OCTA has also been used to investigate the microvascular abnormalities associated with diabetic macular oedema (DMO) [22, 39, 41, 50].

The previously discussed study by Kim et al. which evaluated a number of perfusion indices in eyes with NPDR, performed additional analysis eyes with DMO [39]. Of the 32 mild NPDR eyes imaged using the Cirrus SD-OCTA device, 8 had co-existing DMO. This subgroup had significantly lower vessel density, skeleton density, and fractal dimension in both the SRL and DRL compared to those without DMO [39]. Further longitudinal studies will be required to investigate these associations with $\mathrm{DMO}$ - if these microvascular changes are a risk factor for DMO, or if they occur as a result of DMO. The authors hypothesise that intraretinal fluid may physically displace the vasculature or attenuate the decorrelation signal, or, alternatively, occluded vessels may lead to the development of DMO [39].

Ting et al. [41] demonstrated similar findings using SS-OCTA (Topcon Corp). Of the 100 diabetic eyes imaged, 16 had DMO. Capillary density index was reduced in eyes with DMO compared to those without DMO in both the SCP and DCP; however, in contrast to the indices evaluated by Kim et al., these reductions were not statistically significant (SCP; 0.344 vs. 0.347 , $P=0.16$, and DCP; 0.349 vs. $0.357, P=0.12$ ) [41]. Di et al. [22] analysed the RTVue OCTA images of 113 diabetic eyes, of which 30 had DMO. This group demonstrated that eyes with DMO exhibited increased FAZ areas (non-segmented) compared to those without DMO and that this increase was statistically significant $\left(0.46 \pm 0.12 \mathrm{~mm}^{2}\right.$ vs. $0.40 \pm 0.13 \mathrm{~mm}^{2}$, $P=0.017)$ [22].

Lee et al. [51] investigated the OCTA microvascular features of eyes with DMO, undergoing anti-VEGF therapy. SD-OCT (Spectralis) was used to evaluate the response of DMO eyes to anti-VEGF. A good response was defined as a reduction of $>50 \mu \mathrm{m}$ after three consecutive injections. RTVue OCTA imaging was performed on 103 eyes-51 DMO eyes with a poor response to anti-VEGF, 32 DMO eyes with a good response to anti-VEGF, and 20 control eyes (fellow eyes without a history of DMO) [51]. FAZ area and the number of MAs were increased to a greater extent in DCP compared to SCP in both responders and nonresponders [51]. Vascular flow density was reduced to a greater extent in the DCP compared to SCP in both groups also [51]. DMO eyes with a good response to anti-VEGF were compared to DMO eyes with a poor response. In the DCP, poor responders exhibited larger FAZ areas, higher numbers of MAs, and reduced vascular flow density $(P<0.001$ for each parameter) [51]. There was no significant difference in these parameters in the SCP. In addition, DMO eyes were compared to non-DMO (control) eyes. In the DCP, DMO eyes exhibited larger FAZ areas, more MAs, and reduced vascular flow density $(P<0.001$ for each parameter) [51]. The findings suggest that DCP microvascular integrity may be associated with antiVEGF treatment response, and disease pathogenesis, in eyes with DMO.

De Carlo et al. [52] demonstrated that OCTA has the ability to differentiate between DMO and capillary non-perfusion. The RTVue OCTA images of 17 eyes with DMO were retrospectively analysed. In all cases, OCTA was able to demonstrate cystoid spaces of DMO as areas of complete flow void, appearing as completely black - they demonstrated rounded edges and could therefore be differentiated from the more irregularly delineated capillary non-perfusion areas [52]. Nine eyes from the study were treated with antiVEGF during the period of the study. OCTA demonstrated a corresponding reduction in DMO in all nine cases [52]. The findings are promising in that they suggest OCTA may have a role in the diagnosis of DMO, as well as monitoring the treatment response to anti-VEGF therapy. However, similar to Lee et al., the reliability of current OCTA technology in eyes with DMO was questioned as the presence of DMO resulted in greater difficulty in the segmentation of SCP and DCP [51, 52].

Mane et al. [53] used OCTA to investigate the relationship between capillary non-perfusion and the location of cystoid oedema in DMO. Retrospective analysis was performed on the OCTA (RTVue XR Avanti) images of 20 controls and 24 eyes with chronic diabetic cystoid macular oedema (DCMO). The findings suggest that cystoid spaces are associated with sites of capillary non-perfusion, particularly in the DCP - in the DCMO eyes, cystoid spaces were surrounded by non-perfusion areas in the SCP in $71 \%$ 
of cases, and in the DCP in $96 \%$ of cases [53]. Compared to controls, the mean capillary density was significantly reduced in DCMO eyes, in both plexuses (SCP; $58.43 \pm 2.28 \%$ vs. $44.98 \pm 3.32 \%$, and DCP; $58.84 \pm 2.43 \%$ vs. $51.55 \pm 3.41 \% \quad P<0.001$ for both comparisons) [53]. In contrast to Lee et al., this study noted a greater reduction in perfusion the SCP compared to DCP [51, 53]. However, the authors eluded to the fact that an overestimation on DCP capillary density was likely to have occurred-due to a falsely detected correlation signal from the walls of cystoid spaces and projection artefacts from the SCP [53]. In addition, this study of chronic DCMO cases may not translate to all cases of DCMO.

Alterations in OCTA-based microvascular parameters in response to anti-VEGF have not been extensively researched to date. De Carlo et al. [52] suggested that reappearance of retinal microvasculature may occur following anti-VEGF therapy; however, this has not been definitively demonstrated. In the study by Mane et al. [53], DCMO resolution had occurred in 11 of the cases studied, either spontaneously or after anti-VEGF therapy-and capillary density, in the SCP or DCP, did not significantly change after DCMO resolution. Similarly, Ghasemi et al. [54] found no significant difference in vessel density or FAZ area in eyes with macular oedema before and after anti-VEGF injections. However, limitations of these studies are noted in terms of sample size, and further research is warranted in this area.

Future directions of OCTA in diabetic retinopathy

A number of potential future directions of OCTA technology in DR have been investigated by recent publications. These include additional segmentation analysis [55-57], reduction of projection artefacts [56-58], and swept-source OCTA technology [23, 59-61].

Additional segmentation of retinal vascular layers was investigated in diabetic eyes by Park et al. [55]. Standard OCTA software, as previously described, separates retinal vasculature at the middle of the INL into the SCP and DCP. The middle capillary plexus (MCP) is located at the inner aspect of the INL, therefore it is in incorporated into both these plexuses, mainly the SCP, using this method [55]. This group used the RTVue OCTA device; however, instead of using the built-in segmentation software to visualise the SCP and DCP, they presented a novel method of manual segmentation to evaluate the SCP $(3 \mu \mathrm{m}$ below ILM to the outer IPL), MCP (a $30 \mu \mathrm{m}$ slab from outer IPL boundary), and DCP (a $15 \mu \mathrm{m}$ slab from outer INL, set at $45-60 \mu \mathrm{m}$ below IPL) [55]. Ten control eyes and 12 DR eyes were imaged. Qualitative analysis was performed. In healthy eyes, the MCP appeared to have the most clearly delineated FAZ and a smaller FAZ diameter compared to the SCP and DCP [55]. In diabetic eyes, FAZ disruption and parafoveal non-perfusion occurred to varying extents in the three different plexuses-there was marked disruption of the FAZ outline in the MCP, compared to healthy controls [55]. Further quantitative studies are required in this area.

The reduction of projection artefacts, thereby improving the accuracy of DCP analysis has also been investigated. Hwang et al. used projection-resolved OCTA (PR-OCTA) in eyes with DR and healthy controls [56]. The RTVue OCTA device was used to image 29 control eyes and 47 DR eyes-graded as 8 mild/moderate NPDR, 8 severe NPDR, and 13 PDR. A projection-resolution (PR) algorithm, as described by Zhang et al. [62], was used to correct for projection artefacts. Following this, a semi-automated algorithm was used to segment the images into three distinct vascular plexuses-superficial layer; the inner $80 \%$ of ganglion cell layer, intermediate layer; the outer $20 \%$ of the ganglion cell layer and inner $20 \%$ if INL, and deep layer; half the depth of the INL to outer border of OPL [56]. Three-layer PR-OCTA identified capillary non-perfusion with greater sensitivity than standard two-layer (SCP, DCP) OCTA, 100\% (95\% CI, 90.8-100\%) vs. $78.7 \%$ (95\% CI, 63.9-88.8\%) [56]. It also differentiated severe DR (PDR and severe NPDR) from mild DR (mild/moderate NPDR) with greater sensitivity, $72.2 \%$ (95\% CI, 54.6-85.2\%) vs. $25.0 \%$ (95\% CI, 12.7-42.5\%) [56]. PR-OCTA, and subsequent visualisation of three individual vascular plexuses with reduced projection artefacts in the intermediate and deep layers, may enable improved identification of microvascular abnormalities compared to two-layer OCTA.

This technology was further evaluated by Zhang et al. [57]. PR-OCTA images were obtained for the superficial, intermediate, and deep layers as described by Hwang et al. [56]. This study, however, focused on eyes with mild NPDR-13 control eyes and 13 mild 
NPDR eyes [57]. Non-perfusion was measured in each of the three vascular layers using a novel automated algorithm - the total avascular area (TAA) was calculated in each $3 \mathrm{~mm} \times 3 \mathrm{~mm}$ scan, and the extrafoveal avascular area (EAA) was defined as the TAA excluding a $1 \mathrm{~mm}$ central circle [57]. The EAA from each plexus was summated. The sum of the EAA's from the three plexuses was significantly larger in mild NPDR eyes compared to controls $(P<0.001)$ and had a sensitivity for DR detection of $94 \%($ AROC $=0.99)$ [57]. The results indicate that quantification of avascular area in these three vascular layers using PROCTA may be useful in the early detection of DR.

Swept-source OCTA technology may also offer enhanced investigation of microvascular abnormalities in DR. Studies have been performed in diabetic eyes using the SS-OCTA (Carl Zeiss Meditec) device [59] and the SS-OCTA (DRI OCT Triton; Topcon) device [23, 41, 61].

A qualitative by Matsunaga et al. [59] used the SSOCTA (Carl Zeiss Meditec) device to image 47 eyes with DR. Phase-contrast and intensity-contrast algorithms used to construct three retinal slabs; inner retina (ILM to superficial IPL), middle retina (deep IPL to superficial ONL), and deep retina (deep ONL to ELM) [59]. Like SD-OCTA, SS-OCTA demonstrated the ability to identify previously described microvascular abnormalities such as microaneurysms, capillary nonperfusion and NVD, but also identified additional abnormalities such as IRMA and cotton wool spots [59]. In many cases, SS-OCTA enabled enhanced delineation of the non-perfusion areas, particularly in the inner and middle retina layers [59].

Choi et al. [60] imaged diabetic eyes, with and without retinopathy, using a prototype ultrahigh-speed SS-OCTA device with a light source wavelength of $1050 \mathrm{~nm}$ and $400 \mathrm{kHz}$ scan rate. This prospective study consisted of 63 normal eyes, 51 NDR eyes, 29 NPDR eyes, and 9 PDR eyes [60]. In eyes with DR, reduced capillary density, capillary dropout, and FAZ irregularities were observed [60]. Choriocapillaris flow impairment was noted in 7 PDR eyes and 15 NPDR eyes [60]. In addition to visualisation of the retinal vascular layers, ultrahigh-speed SS-OCTA technology enables deeper tissue penetration, allowing more accurate analysis of the choriocapillaris [60]. With faster image acquisition speeds, the technology may also permit wider field imaging [60].
An improved method for detection of blood flow has recently been described. The SSADA algorithm, as used in conventional SD-OCTA technology, characterises vasculature by detecting the presence of blood flow [4]. In addition to visualising the microvasculature, a novel variable interscan time analysis (VISTA) algorithm has demonstrated the ability to evaluate the speed of the detected blood flow [63, 64]. The technology has been implemented in combination with a vertical cavity surface emitting laser (VCSEL) swept light source OCTA prototype, with promising results $[63,64]$. It is hoped that this technology will improve the quantitative interpretation of OCTA.

\section{Conclusion}

OCTA can identify microaneurysms (MAs) in eyes with DR, allowing enhanced analysis compared to FA, in that their intraretinal location can be identifiedthat is SCP or DCP [4, 6]. A significantly higher number of MAs occur in the DCP, compared to the SCP $[6,11]$. DCP MAs also appear to be significantly associated with areas of DMO [10]. However, the sensitivity for MA detection is a limitation of current OCTA technology [6]. It is hypothesised that blood flow in MAs below a threshold of $0.3 \mathrm{~mm}$ per second is not detected by the SSADA algorithm [7]. With advancing technology, this may improve-ultrahighspeed SS-OCTA has been shown to have a sensitivity of $85 \%$ and a specificity of $75 \%$ for MA detection compared to FA [12].

By adjusting the segmentation boundary to detect blood flow above the internal limiting membrane (ILM), OCTA can visualise preretinal neovascularisation in eyes with proliferative DR [4]. OCTA can characterise the extent of NVD with greater accuracy than FA-due to the absence of obscuration of its outline with fluorescein dye leakage [14]. Enhanced delineation of NVD can potentially enable quantitative assessment of neovascularisation, whereby its area can be precisely measured [14]. OCTA can also identify areas of capillary non-perfusion associated with the neovascularisation [16]. Furthermore, it may enable a morphological classification on NV, based on the presence or absence of exuberant vascular proliferation (EVP) - a potential indicator of disease activity for monitoring of treatment [17]. However, in its present form, the restricted field of view is a significant 
drawback of OCTA imaging, in that peripheral neovascularisation cannot be evaluated [16].

The foveal avascular zone has been one of the most extensively investigated areas in DR using OCTA. Eyes of diabetic patients, even without retinopathy (NDR), have been shown to have significantly enlarged FAZ areas compared to controls-in both the SCP and DCP [19]. However, inconsistencies exist in the literature. Dimitrova et al. found that the FAZ area was significantly increased in NDR eyes compared to controls in the SCP, but the increase was not significant in the DCP [20]. OCTA has also demonstrated the ability to differentiate between different subgroups of DR severity-Al-Sheikh et al. found significant differences in FAZ area between all DR subgroups at the level of the DCP, but just between the severe NPDR and PDR subgroups at the level of the SCP [23]. A number of OCTA devices and segmentation methods have been used to date (Table 1). In addition, the aforementioned inaccuracies in interpretation of the deeper vascular networks due to projection artefact [38] mean it is difficult to draw conclusions on the diagnostic value of FAZ area in DR at present. Further FAZ parameters of acircularity index, axis ratio, FAZ perimeter, radius, and diameter have also shown promise as markers of DR on OCTA imaging [22, 35, 36, 65].

OCTA-based measurements have been used as perfusion indices in eyes with DR (Table 2). Several OCTA devices have been used, as well as different segmentation methods (Table 2). The region in which perfusion is evaluated has also varied between studies-some analysing the entire $3 \mathrm{~mm} \times 3 \mathrm{~mm}$ or $6 \mathrm{~mm} \times 6 \mathrm{~mm}$ scan area, others analysing a parafoveal or perifoveal area. Furthermore, the definition of these para-/perifoveal areas has differed between studies [20, 28-30]. This makes overall interpretation of this data more difficult. Nonetheless, there is significant evidence that the density of vascular perfusion reduces with increasing severity of DR and that OCTA parameters can be used to identify these changes (Table 2). It can also be used to detect early DR. Capillary perfusion density (CPD) has been shown to be significantly lower in eyes with mild NPDR compared to controls, in both the SCP and DCP [37]. It has also been demonstrated that vessel density is significantly reduced in NDR eyes compared to controls, in both the SCP and DCP [20]. However, as with FAZ measurements, there have been inconsistencies noted in the literature, particularly in relation to deep plexus analysis [20, 23, 28].

OCTA enables evaluation of the branching complexity of vessels in DR-in the form of fractal dimension (FD) measurements. FD has been shown to be significantly reduced in DR eyes compared to controls [42]. It can be used to differentiate mild NPDR eyes from controls, based on SCP analysis [39]. Measurement of vessel spacing and vessel tortuosity using OCTA has also been proposed as methods of analysing DR severity [33, 34].

Compared to FA, OCTA has demonstrated moderate agreement for grading of DMI [45]. Using OCTA, areas of macular non-perfusion can be accurately delineated and measured in both the SCP and DCP [4]. As a result of this, and the absence of fluorescein dye leakage, OCTA may enable enhanced analysis of nonperfusion [6]. However, the small field of view obtained is a limitation with current technology, meaning that peripheral retinal non-perfusion cannot be seen [4]. Nevertheless, OCTA is showing promising results for the evaluation capillary non-perfusion and particularly automated methods of analysis [29].

OCTA has also been used to investigate microvascular abnormalities associated with diabetic macular oedema (DMO). Kim et al. [39] demonstrated that DR eyes with DMO have significantly reduced vessel density, skeleton density, and fractal dimension, compared to DR eyes without DMO. OCTA is improving our understanding of the role of the DCP in the pathogenesis of DMO. In eyes with DMO, FAZ area and the number of MAs appear to be increased to a greater extent in the DCP compared to the SCP [51]. In addition, flow density appears to be reduced to a greater extent in the DCP [51]. The ability to simultaneously obtain structural OCT and OCTA images may be of benefit for monitoring of treatment response in DMO. It has been demonstrated that poor responders to anti-VEGF have significantly larger FAZ areas, more MAs, and reduced flow density in the DCP, compared to good-responders [51].

OCTA technology is evolving, and the future directions of OCTA are becoming apparent. Additional segmentation analysis, and evaluation of the middle capillary plexus, may further enhance the ability of OCTA to detect early microvascular changes in DR [55-57]. Projection-resolution algorithms have been developed to reduce projection artefact and thereby improve the accuracy of analysis of the middle 
and deep vascular layers [62]. Ultrahigh-speed sweptsource OCTA technology may also offer improved microvasculature analysis, enhanced depth penetration and choriocapillaris visualisation, and wide-field OCTA imaging [59, 60].

In conclusion, OCTA has demonstrated the ability to accurately visualise retinal microvasculature in eyes with diabetic retinopathy. It has the potential to quantitatively analyse neovascularisation, capillary non-perfusion, and diabetic macular ischaemia, in addition to novel vascular parameters such as fractal dimension, vessel tortuosity, and skeleton density. It can detect early microvascular changes, in eyes with or without clinically evident DR. It has been shown to detect progressive changes in the foveal avascular zone, and vascular perfusion density, with worsening severity of disease. By enabling three-dimensional visualisation of the individual retinal vascular networks, OCTA is enhancing our understanding of the role of the deeper vasculature in the pathogenesis of diabetic retinopathy and maculopathy. However, limitations exist with current OCTA technology, in respect to the small field of view, projection artefact, and inaccuracies in analysis of the deeper vascular layers. While questions remain regarding its practical applicability in its current form, with continuing development and improvement of the technology, the diagnostic value of OCTA in diabetic retinopathy is likely to become evident.

\section{Compliance with ethical standards}

Conflict of interest All authors certify that they have no affiliations with or involvement in any organisation or entity with any financial interest (such as honoraria; educational grants; participation in speakers' bureaus; membership, employment, consultancies, stock ownership, or other equity interest; and expert testimony or patent-licensing arrangements), or non-financial interest (such as personal or professional relationships, affiliations, knowledge, or beliefs) in the subject matter or materials discussed in this manuscript.

Open Access This article is distributed under the terms of the Creative Commons Attribution 4.0 International License (http:// creativecommons.org/licenses/by/4.0/), which permits unrestricted use, distribution, and reproduction in any medium, provided you give appropriate credit to the original author(s) and the source, provide a link to the Creative Commons license, and indicate if changes were made.

\section{References}

1. Klein R, Klein BE, Moss SE, Davis MD, DeMets DL (1984) The Wisconsin epidemiologic study of diabetic retinopathy. III. Prevalence and risk of diabetic retinopathy when age at diagnosis is 30 or more years. Arch Ophthalmol (Chicago, Ill 1960) 102(4):527-532

2. Saaddine JB, Honeycutt AA, Narayan KMV, Zhang X, Klein R, Boyle JP (2008) Projection of diabetic retinopathy and other major eye diseases among people with diabetes mellitus: United States, 2005-2050. Arch Ophthalmol (Chicago, Ill 1960) 126(12):1740-1747

3. Yannuzzi LA, Rohrer KT, Tindel LJ, Sobel RS, Costanza MA, Shields W et al (1986) Fluorescein angiography complication survey. Ophthalmology 93(5):611-617

4. Ishibazawa A, Nagaoka T, Takahashi A, Omae T, Tani T, Sogawa K et al (2015) Optical coherence tomography angiography in diabetic retinopathy: a prospective pilot study. Am J Ophthalmol 160(1):35-44

5. Moher D, Shamseer L, Clarke M, Ghersi D, Liberati A, Petticrew M et al (2015) Preferred reporting items for systematic review and meta-analysis protocols (PRISMA-P) 2015 statement. Syst Rev 4:1

6. Couturier A, Mane V, Bonnin S, Erginay A, Massin P, Gaudric A et al (2015) Capillary plexus anomalies in diabetic retinopathy on optical coherence tomography angiography. Retina 35(11):2384-2391

7. Tokayer J, Jia Y, Dhalla A-H, Huang D (2013) Blood flow velocity quantification using split-spectrum amplitudedecorrelation angiography with optical coherence tomography. Biomed Opt Express 4(10):1909-1924

8. Parravano M, De Geronimo D, Scarinci F, Querques L, Virgili G, Simonett JM et al (2017) Relationship between internal reflectivity of diabetic microaneurysms on SD-OCT and detection on OCT angiography. Am J Ophthalmol 179:90-96

9. Miwa Y, Murakami T, Suzuma K, Uji A, Yoshitake S, Fujimoto M et al (2016) Relationship between functional and structural changes in diabetic vessels in optical coherence tomography angiography. Sci Rep 6:29064

10. Hasegawa N, Nozaki M, Takase N, Yoshida M, Ogura Y (2016) New insights into microaneurysms in the deep capillary plexus detected by optical coherence tomography angiography in diabetic macular edema. Invest Ophthalmol Vis Sci 57(9):OCT348-OCT355

11. Peres MB, Kato RT, Kniggendorf VF, Cole ED, Onal S, Torres E et al (2016) Comparison of optical coherence tomography angiography and fluorescein angiography for the identification of retinal vascular changes in eyes with diabetic macular edema. Ophthalmic Surg Lasers Imaging Retina 47(11):1013-1019

12. Salz DA, de Carlo TE, Adhi M, Moult E, Choi W, Baumal CR et al (2016) Select features of diabetic retinopathy on swept-source optical coherence tomographic angiography compared with fluorescein angiography and normal eyes. JAMA Ophthalmol 134(6):644-650

13. Jia Y, Bailey ST, Hwang TS, McClintic SM, Gao SS, Pennesi ME et al (2015) Quantitative optical coherence tomography angiography of vascular abnormalities in the 
living human eye. Proc Natl Acad Sci U S A 112(18):E2395-E2402

14. Savastano MC, Federici M, Falsini B, Caporossi A, Minnella AM (2016) Detecting papillary neovascularization in proliferative diabetic retinopathy using optical coherence tomography angiography. Acta Ophthalmol. 30:353-359

15. Zhang M, Wang J, Pechauer AD, Hwang TS, Gao SS, Liu L et al (2015) Advanced image processing for optical coherence tomographic angiography of macular diseases. Biomed Opt Express 6(12):4661-4675

16. de Carlo TE, Bonini Filho MA, Baumal CR, Reichel E, Rogers A, Witkin AJ et al (2016) Evaluation of preretinal neovascularization in proliferative diabetic retinopathy using optical coherence tomography angiography. Ophthalmic Surg Lasers Imaging Retina 47(2):115-119

17. Ishibazawa A, Nagaoka T, Yokota H, Takahashi A, Omae T, Song Y-S et al (2016) Characteristics of retinal neovascularization in proliferative diabetic retinopathy imaged by optical coherence tomography angiography. Invest Ophthalmol Vis Sci 57(14):6247-6255

18. Singh A, Agarwal A, Mahajan S, Karkhur S, Singh R, Bansal R et al (2017) Morphological differences between optic disc collaterals and neovascularization on optical coherence tomography angiography. Graefes Arch Clin Exp Ophthalmol 255(4):753-759

19. Takase N, Nozaki M, Kato A, Ozeki H, Yoshida M, Ogura Y (2015) Enlargement of foveal avascular zone in diabetic eyes evaluated by en face optical coherence tomography angiography. Retina 35(11):2377-2383

20. Dimitrova G, Chihara E, Takahashi H, Amano H, Okazaki K (2017) Quantitative retinal optical coherence tomography angiography in patients with diabetes without diabetic retinopathy. Invest Ophthalmol Vis Sci 58(1):190-196

21. de Carlo TE, Chin AT, Bonini Filho MA, Adhi M, Branchini L, Salz DA et al (2015) Detection of microvascular changes in eyes of patients with diabetes but not clinical diabetic retinopathy using optical coherence tomography angiography. Retina 35(11):2364-2370

22. Di G, Weihong Y, Xiao Z, Zhikun Y, Xuan Z, Yi Q et al (2016) A morphological study of the foveal avascular zone in patients with diabetes mellitus using optical coherence tomography angiography. Graefes Arch Clin Exp Ophthalmol 254(5):873-879

23. Al-Sheikh M, Akil H, Pfau M, Sadda SR (2016) SweptSource OCT angiography imaging of the foveal avascular zone and macular capillary network density in diabetic retinopathy. Invest Ophthalmol Vis Sci 57(8):3907-3913

24. Gozlan J, Ingrand P, Lichtwitz O, Cazet-Supervielle A, Benoudis L, Boissonnot M et al (2017) Retinal microvascular alterations related to diabetes assessed by optical coherence tomography angiography: a cross-sectional analysis. Medicine (Baltimore) 96(15):e6427

25. Balaratnasingam $\mathrm{C}$, Inoue $\mathrm{M}$, Ahn S, McCann J, DhramiGavazi E, Yannuzzi LA et al (2016) Visual acuity is correlated with the area of the foveal avascular zone in diabetic retinopathy and retinal vein occlusion. Ophthalmology 123(11):2352-2367

26. Yu S, Lu J, Cao D, Liu R, Liu B, Li T et al (2016) The role of optical coherence tomography angiography in fundus vascular abnormalities. BMC Ophthalmol 16:107
27. Samara WA, Shahlaee A, Adam MK, Khan MA, Chiang A, Maguire JI et al (2017) Quantification of diabetic macular ischemia using optical coherence tomography angiography and its relationship with visual acuity. Ophthalmology 124(2):235-244

28. Simonett JM, Scarinci F, Picconi F, Giorno P, De Geronimo D, Di Renzo A et al (2017) Early microvascular retinal changes in optical coherence tomography angiography in patients with type 1 diabetes mellitus. Acta Ophthalmol 95:e751-e755

29. Hwang TS, Gao SS, Liu L, Lauer AK, Bailey ST, Flaxel CJ et al (2016) Automated quantification of capillary nonperfusion using optical coherence tomography angiography in diabetic retinopathy. JAMA Ophthalmol 134(4):367-373

30. Sambhav K, Abu-Amero KK, Chalam KV (2017) Deep capillary macular perfusion indices obtained with OCT angiography correlate with degree of nonproliferative diabetic retinopathy. Eur J Ophthalmol 27:716-729

31. Durbin MK, An L, Shemonski ND, Soares M, Santos T, Lopes M et al (2017) Quantification of retinal microvascular density in optical coherence tomographic angiography images in diabetic retinopathy. JAMA Ophthalmol 135(4):370-376

32. Carnevali A, Sacconi R, Corbelli E, Tomasso L, Querques L, Zerbini G et al (2017) Optical coherence tomography angiography analysis of retinal vascular plexuses and choriocapillaris in patients with type 1 diabetes without diabetic retinopathy. Acta Diabetol 54:695-702

33. Bhanushali D, Anegondi N, Gadde SGK, Srinivasan P, Chidambara L, Yadav NK et al (2016) Linking retinal microvasculature features with severity of diabetic retinopathy using optical coherence tomography angiography. Invest Ophthalmol Vis Sci 57(9):OCT519-OCT525

34. Lee H, Lee M, Chung H, Kim HC (2017) Quantification of retinal vessel tortuosity in diabetic retinopathy using optical coherence tomography angiography. Retina 38:1

35. Krawitz BD, Mo S, Geyman LS, Agemy SA, Scripsema NK, Garcia PM et al (2017) Acircularity index and axis ratio of the foveal avascular zone in diabetic eyes and healthy controls measured by optical coherence tomography angiography. Vis Res 139:177-186

36. Freiberg FJ, Pfau M, Wons J, Wirth MA, Becker MD, Michels S (2016) Optical coherence tomography angiography of the foveal avascular zone in diabetic retinopathy. Graefes Arch Clin Exp Ophthalmol 254(6):1051-1058

37. Agemy SA, Scripsema NK, Shah CM, Chui T, Garcia PM, Lee JG et al (2015) Retinal vascular perfusion density mapping using optical coherence tomography angiography in normals and diabetic retinopathy patients. Retina 35(11):2353-2363

38. Spaide RF, Fujimoto JG, Waheed NK (2015) Image artifacts in optical coherence tomography angiography. Retina 35(11):2163-2180

39. Kim AY, Chu Z, Shahidzadeh A, Wang RK, Puliafito CA, Kashani AH (2016) Quantifying microvascular density and morphology in diabetic retinopathy using spectral-domain optical coherence tomography angiography. Invest Ophthalmol Vis Sci 57(9):OCT362-OCT370

40. You Q, Freeman WR, Weinreb RN, Zangwill L, Manalastas PIC, Saunders LJ et al (2016) Reproducibility of vessel density measurement with optical coherence tomography 
angiography in eyes with and without retinopathy. Retina 37(8):1475-1482

41. Ting DSW, Tan GSW, Agrawal R, Yanagi Y, Sie NM, Wong CW et al (2017) Optical coherence tomographic angiography in type 2 diabetes and diabetic retinopathy. JAMA Ophthalmol 135(4):306-312

42. Zahid S, Dolz-Marco R, Freund KB, Balaratnasingam C, Dansingani K, Gilani F et al (2016) Fractal dimensional analysis of optical coherence tomography angiography in eyes with diabetic retinopathy. Invest Ophthalmol Vis Sci 57(11):4940-4947

43. Minnella AM, Savastano MC, Federici M, Falsini B, Caporossi A (2016) Superficial and deep vascular structure of the retina in diabetic macular ischaemia: OCT angiography. Acta Ophthalmol 96(5):e647-e648

44. Scarinci F, Nesper PL, Fawzi AA (2016) Deep retinal capillary nonperfusion is associated with photoreceptor disruption in diabetic macular ischemia. Am J Ophthalmol 168:129-138

45. Bradley PD, Sim DA, Keane PA, Cardoso J, Agrawal R, Tufail A et al (2016) The evaluation of diabetic macular ischemia using optical coherence tomography angiography. Invest Ophthalmol Vis Sci 57(2):626-631

46. Hwang TS, Jia Y, Gao SS, Bailey ST, Lauer AK, Flaxel CJ et al (2015) Optical coherence tomography angiography features of diabetic retinopathy. Retina 35(11):2371-2376

47. Soares M, Neves C, Marques IP, Pires I, Schwartz C, Costa MA et al (2017) Comparison of diabetic retinopathy classification using fluorescein angiography and optical coherence tomography angiography. $\mathrm{Br} \mathrm{J}$ Ophthalmol 101(1):62-68

48. Cennamo G, Romano MR, Nicoletti G, Velotti N, de Crecchio G (2017) Optical coherence tomography angiography versus fluorescein angiography in the diagnosis of ischaemic diabetic maculopathy. Acta Ophthalmol 95(1):e36-e42

49. Early Treatment Diabetic Retinopathy Study Research Group (1991) Classification of diabetic retinopathy from fluorescein angiograms. ETDRS report number 11. Ophthalmology 98(5 Suppl):807-822

50. Mao L, Weng S-S, Gong Y-Y, Yu S-Q (2017) Optical coherence tomography angiography of macular telangiectasia type 1: comparison with mild diabetic macular edema. Lasers Surg Med 49(3):225-232

51. Lee J, Moon BG, Cho AR, Yoon YH (2016) Optical coherence tomography angiography of DME and its association with anti-VEGF treatment response. Ophthalmology 123(11):2368-2375

52. de Carlo TE, Chin AT, Joseph T, Baumal CR, Witkin AJ, Duker JS et al (2016) Distinguishing diabetic macular edema from capillary nonperfusion using optical coherence tomography angiography. Ophthalmic Surg Lasers Imaging Retina 47(2):108-114

53. Mane V, Dupas B, Gaudric A, Bonnin S, Pedinielli A, Bousquet E et al (2016) Correlation between cystoid spaces in chronic diabetic macular edema and capillary nonperfusion detected by optical coherence tomography angiography. Retina 36:S102-S110
54. Ghasemi Falavarjani K, Iafe NA, Hubschman J-P, Tsui I, Sadda SR, Sarraf D (2017) Optical coherence tomography angiography analysis of the foveal avascular zone and macular vessel density after anti-VEGF therapy in eyes with diabetic macular edema and retinal vein occlusion. Invest Ophthalmol Vis Sci 58(1):30-34

55. Park JJ, Soetikno BT, Fawzi AA (2016) Characterization of the middle capillary plexus using optical coherence tomography angiography in healthy and diabetic eyes. Retina 36(11):2039-2050

56. Hwang TS, Zhang M, Bhavsar K, Zhang X, Campbell JP, Lin $P$ et al (2016) Visualization of 3 distinct retinal plexuses by projection-resolved optical coherence tomography angiography in diabetic retinopathy. JAMA Ophthalmol 134(12):1411-1419

57. Zhang M, Hwang TS, Dongye C, Wilson DJ, Huang D, Jia Y (2016) Automated quantification of nonperfusion in three retinal plexuses using projection-resolved optical coherence tomography angiography in diabetic retinopathy. Invest Ophthalmol Vis Sci 57(13):5101-5106

58. Dongye C, Zhang M, Hwang TS, Wang J, Gao SS, Liu L et al (2017) Automated detection of dilated capillaries on optical coherence tomography angiography. Biomed Opt Express 8(2):1101-1109

59. Matsunaga DR, Yi JJ, De Koo LO, Ameri H, Puliafito CA, Kashani AH (2015) Optical coherence tomography angiography of diabetic retinopathy in human subjects. Ophthalmic Surg Lasers Imaging Retina 46(8):796-805

60. Choi W, Waheed NK, Moult EM, Adhi M, Lee B, De Carlo $\mathrm{T}$ et al (2017) Ultrahigh speed swept source optical coherence tomography angiography of retinal and choriocapillaris alterations in diabetic patients with and without retinopathy. Retina 37(1):11-21

61. Stanga PE, Papayannis A, Tsamis E, Stringa F, Cole T, D'Souza Y et al (2016) New findings in diabetic maculopathy and proliferative disease by swept-source optical coherence tomography angiography. Dev Ophthalmol 56:113-121

62. Zhang M, Hwang TS, Campbell JP, Bailey ST, Wilson DJ, Huang D et al (2016) Projection-resolved optical coherence tomographic angiography. Biomed Opt Express 7(3):816-828

63. Ploner SB, Moult EM, Choi W, Waheed NK, Lee B, Novais EA et al (2016) Toward quantitative optical coherence tomography angiography: visualizing blood flow speeds in ocular pathology using variable interscan time analysis. Retina 36(Suppl 1):S118-S126

64. Schottenhamml J, Moult EM, Ploner S, Lee B, Novais EA, Cole E et al (2016) An automatic, intercapillary area-based algorithm for quantifying diabetes-related capillary dropout using optical coherence tomography angiography. Retina 36(Suppl 1):S93-S101

65. Mo S, Krawitz B, Efstathiadis E, Geyman L, Weitz R, Chui TYP et al (2016) Imaging foveal microvasculature: optical coherence tomography angiography versus adaptive optics scanning light ophthalmoscope fluorescein angiography. Invest Ophthalmol Vis Sci. 57(9):OCT130-OCT140 